\title{
Intervençáo Comportamental Precoce e Intensiva com Crianças com Autismo por Meio da Capacitação de Cuidadores ${ }^{1}$ \\ EARLY AND INTENSIVE BEHAVIORAL INTERVENTION WITH CHILDREN WITH AUTISM Through CAREGIVER TRAINING
}

\author{
Camila Graciella Santos GOMES² \\ Deisy das Graças de SOUZA ${ }^{3}$ \\ Analice Dutra SILVEIRA ${ }^{4}$ \\ Ianaiara Marprates OLIVEIRA ${ }^{5}$
}

\begin{abstract}
RESUMO: o estudo avaliou os efeitos do primeiro ano de Intervenção Comportamental Intensiva no desenvolvimento de 9 crianças com autismo, com idades entre 1 ano e 3 meses e 2 anos e 11 meses, atendidas por um centro brasileiro especializado. A intervenção, conduzida por aproximadamente 15 horas semanais, foi realizada na residência dos participantes por meio da capacitação dos cuidadores. As crianças foram avaliadas pelo Psychoeducational Profile-Revised - PEP-R e pelo Inventário Portage Operacionalizado- IPO, no início e ao término da intervençấo. Os dados foram analisados individualmente e os resultados indicaram ganhos no desenvolvimento de todas as crianças, embora em graus variados.
\end{abstract}

PALAVRAS-CHAVE: Educação Especial. Autismo. Análise Comportamental. Estimulação Precoce. Cuidador.

\begin{abstract}
The study evaluated the effects of the first year of Intensive Behavioral Intervention on the development of 9 children with autism, aged between 1 year and 3 months and 2 years and 11 months, served by a specialized Brazilian center. The intervention, conducted for approximately 15 hours per week, was performed at the participants' homes through the training of caregivers. The children were evaluated through the Psychoeducational Profile-Revised and the Inventário Portage Operacionalizado, at the beginning and at the end of the intervention. Data were analyzed individually and the results indicated gains in the development of all children, although in varying degrees.
\end{abstract}

KEYWORDS: Special Education. Autism. Behavior Analysis. Early Stimulation. Caregiver.

\section{INTRODUÇÃo}

O autismo é um transtorno do desenvolvimento infantil caracterizado por alteraçóes nas interações sociais e na comunicação e pela presença de interesses restritos, fixos e intensos e comportamentos repetitivos (AMERICAN PSYCHIATRIC ASSOCIATION [APA], 2013). Até o momento não há cura para o transtorno (APA, 2013; GARGIULO, 2006), porém Intervençóes Comportamentais Intensivas têm promovido ganhos significativos no desenvolvimento de crianças com esse diagnóstico desde a década de 1980 (BOYD; CORLEY, 2001; CAMPBELL et al., 1987; DAWSON et al., 2010; LOVAAS, 1987; SMITH, 1999; VIRUÉS-

\footnotetext{
${ }^{1}$ http://dx.doi.org/10.1590/S1413-65382317000300005

${ }^{2}$ Diretora do CEI Desenvolvimento Humano, Docente da Faculdade de Ciências Médicas de Minas Gerais e membro do Instituto Nacional de Ciência e Tecnologia sobre Comportamento, Cognição e Ensino. camila.gomes@ceidesenvolvimentohumano.com.br

${ }^{3}$ Docente do Programa de Pós-Graduação em Educação Especial, Universidade Federal de São Carlos. Centro de Educação e Ciências Humanas. Laboratório de Estudos do Comportamento Humano. Membro do Instituto Nacional de Ciência e Tecnologia sobre Comportamento, Cognição e Ensino. deisydesouza@gmail.com

${ }^{4}$ Diretora do CEI Desenvolvimento Humano. analicesilveira2013@gmail.com

${ }^{5}$ Psicóloga do CEI Desenvolvimento Humano. ianaiaraprates@gmail.com
} 
ORTEGA, 2010; WARREN et al., 2011). Considera-se como "Intervenção Comportamental Intensiva” os modelos de intervenção compostos por estimulação individualizada (um educador para uma criança com autismo), realizados por 15 a 40 horas semanais, por pelo menos dois anos consecutivos, que abrangem várias áreas do desenvolvimento simultaneamente e que são fundamentados em princípios de Análise do Comportamento (GREEN, 1996), caracterizados como Análise do Comportamento Aplicada (Applied Behavior Analysis-ABA; BAER; WOLF; RISLEY, 1987).

A Análise do Comportamento é uma ciência que se interessa pelo estudo das variáveis que afetam os comportamentos (TODOROV; HANNA, 2010). A aplicação dos princípios dessa ciência para a resolução de demandas socialmente relevantes é chamada de Análise do Comportamento Aplicada (BAER; WOLF; RISLEY, 1987) e não ocorre exclusivamente na área do autismo, mas em âmbitos diversos, como na clínica psicológica, na educação, na economia, no desempenho esportivo, entre outros. Tipicamente é feita uma análise da função dos comportamentos que são relevantes e das condiçóes de ensino, em termos de arranjos ambientais e de variáveis motivacionais (consequências reforçadoras) para promover o desenvolvimento desses comportamentos (se ausentes) e seu fortalecimento, se eles ainda ocorrem de maneira incipiente (SKINNER, 1968). Apesar da aplicação da Análise do Comportamento ocorrer em áreas diversas, observa-se um crescimento mais expressivo desse tipo de intervenção na área do autismo, especialmente no formato de Intervenção Comportamental Intensiva (VIRUÉS-ORTEGA, 2010).

O primeiro estudo sobre Intervenção Comportamental Intensiva aplicada ao tratamento do autismo foi publicado na década de 1980 (LOVAAS, 1987). Nesse trabalho três grupos de crianças com autismo, que tinham idades abaixo de 4 anos no início do estudo, foram avaliados. $\mathrm{O}$ grupo experimental, composto por 19 crianças, foi exposto à Intervenção Comportamental Intensiva, com um educador para cada criança, por 40 horas semanais ou mais, por dois ou mais anos consecutivos. O tratamento consistiu no ensino simultâneo de habilidades em diversas áreas (ex. comunicação, interação social, imitação, autocuidados) visando melhorar o desenvolvimento das crianças. Um grupo controle, composto por 19 crianças, recebeu intervenção comportamental mínima, por 10 h semanais ou menos; um segundo grupo controle era composto por 21 participantes que foram tratados em outros centros de atendimento que não realizavam intervenção comportamental ou outro tipo de intervenção intensiva. Os resultados indicaram que $47 \%$ das crianças expostas à Intervenção Comportamental Intensiva tiveram redução significativa dos sintomas de autismo, apresentando desenvolvimento próximo ao esperado para a idade cronológica (próximo ao de uma criança típica, sem autismo); 42\% tiveram uma redução acentuada dos sintomas e 11\% continuaram com sintomas graves de autismo. Crianças do grupo controle que receberam intervençáo comportamental mínima, obtiveram resultados muito diferentes; $2 \%$ apresentaram desenvolvimento próximo ao típico, 45\% tiveram uma redução dos sintomas e 53\% continuaram com sintomas graves de autismo. As crianças do outro grupo controle, que foram tratadas em outros centros de atendimento que não realizavam intervenção comportamental ou intensiva, também apresentaram resultados muito aquém dos obtidos pelas crianças do grupo experimental. Os dados gerais indicaram que aproximadamente $90 \%$ das crianças do projeto apresentaram melhora no desenvolvimento e metade das crianças apresentou desenvolvimento próximo ao típico. Em estudo 
subsequente, realizado quando as crianças tinham aproximadamente 12 anos, constatou-se a manutenção desses resultados (MCEACHIN; SMITH; LOVAAS, 1993).

Os resultados do estudo do Lovaas (1987) foram parcialmente replicados por dezenas de outros estudos posteriores, que utilizaram educadores diferentes (estudantes, profissionais e familiares) e contextos múltiplos (instituições especializadas, escolas e residência das crianças), e que indicaram, no geral, melhora no desenvolvimento dos participantes após realizarem a Intervenção Comportamental Intensiva, inclusive quando comparados a outros tipos de terapias não intensivas ou não fundamentadas na Análise do Comportamento. Esses estudos descreverem resultados variados em relação à proporção dos ganhos da intervenção, mas de modo geral indicaram ganhos no desenvolvimento, especialmente quando realizada precocemente (ELDEVIK et al., 2009; SMITH; BUCH; GAMBY, 2000; WARREN et al., 2011). Apesar da indicação consistente da eficácia de intervenções precoces e intensivas fundamentadas em Análise do Comportamento para o tratamento do autismo, não há, até o momento, estudos publicados indicando efeitos desse tipo de intervenção com crianças brasileiras com autismo.

Nesse contexto, o objetivo desse estudo foi avaliar os efeitos da Intervenção Comportamental Intensiva, realizada precocemente por meio de cuidadores especialmente capacitados para a tarefa, no desenvolvimento de crianças brasileiras com autismo. A pesquisa foi realizada a partir dos dados de crianças que foram atendidas por um centro brasileiro especializado com metodologia semelhante à empregada em outros estudos que utilizaram dados produzidos por centros especializados (LUISELLI et al., 2000; HARRIS; HANDLEMAN, 2000; WEISS, 1999). O desempenho dos participantes foi analisado individualmente a partir de avaliação realizada antes e após a intervenção, utilizando instrumentos padronizados que permitiram medir o desenvolvimento das crianças (por área e no global) e compará-lo em relação ao que era esperado para a idade cronológica. Foram analisados os dados do primeiro ano de Intervenção Comportamental Intensiva.

\section{MéTodo}

\subsection{Participantes}

Participaram do estudo 9 crianças, com idades entre 1 ano e 3 meses e 2 anos e 11 meses, e seus respectivos cuidadores, que foram atendidos pelo Centro de Estudos e Intervenção para o Desenvolvimento Humano (CEI) entre 2011 e 2016 (Tabela 1). Todas as crianças tinham o diagnóstico prévio de autismo (P3, P4, P5, P6, P7, P8, P9) ou suspeita de autismo (P1 e P2), sem o diagnóstico de comorbidades, realizado por psiquiatra ou neurologista infantil especializados, independentes do CEI, seguindo critérios da CID-10 (OMS, 1993), do DSMIV-TR (APA, 2002) ou do DSM-V (APA, 2013). Uma criança apresentava fala (P7) e oito não falavam (P1, P2, P3, P4, P5, P6, P8, P9) antes do início da intervenção. Os cuidadores principais eram estagiárias de psicologia (P1, P2, P4) e pedagogia (P5), mães (P3, P8, P9), pais (P7) e babá (P6). 
Tabela 1 - Características Gerais dos Participantes

Participantes, Idade Cronológica, Intervalo em Meses entre as Avaliaçóes, Presença de Fala, Cuidador e Número de Programas de Habilidades Básicas

\begin{tabular}{|c|c|c|c|c|c|c|c|}
\hline \multirow{2}{*}{ Participante } & \multicolumn{2}{|c|}{ Idade } & \multirow{2}{*}{ Meses } & \multicolumn{2}{|c|}{ Fala } & \multirow{2}{*}{ Cuidador } & \multirow{2}{*}{ Programa } \\
\hline & Pré & Pós & & Pré & Pós & & \\
\hline P1 & 1a $3 \mathrm{~m}$ & 1a $11 \mathrm{~m}$ & 8 & não & sim & estagiária & 28 \\
\hline P2 & 1a $3 \mathrm{~m}$ & 2a $3 \mathrm{~m}$ & 12 & não & sim & estagiária & 13 \\
\hline $\mathbf{P 3}$ & 1a $10 \mathrm{~m}$ & 2a $11 \mathrm{~m}$ & 12 & não & não & mãe & 18 \\
\hline P4 & 1a $11 \mathrm{~m}$ & 2a $11 \mathrm{~m}$ & 12 & não & não & estagiária & 16 \\
\hline P5 & $2 a$ & $3^{\mathrm{a}}$ & 12 & não & sim & estagiária & 21 \\
\hline P6 & $2 \mathrm{a} 2 \mathrm{~m}$ & $3 a 3 m$ & 13 & não & não & babá & 17 \\
\hline P7 & $2 \mathrm{a} 8 \mathrm{~m}$ & $3 \mathrm{a} 8 \mathrm{~m}$ & 12 & $\operatorname{sim}$ & $\operatorname{sim}$ & pais & 16 \\
\hline P8 & 2a $10 \mathrm{~m}$ & 3a $9 \mathrm{~m}$ & 11 & não & $\operatorname{sim}$ & mãe & 17 \\
\hline P9 & 2a $11 \mathrm{~m}$ & 3a $11 \mathrm{~m}$ & 12 & não & sim & mãe & 23 \\
\hline
\end{tabular}

Fonte: elaboração própria.

\subsection{InSTRUMENTOS DE AVALIAÇÁO}

Foram utilizados o Psychoeducational Profile-Revised - PEP-R (SCHOPLER, et al., 1990) e o Inventário Portage Operacionalizado- IPO (WILLIAMS; AIELLO, 2001). Optouse pela utilização desses dois instrumentos por três motivos: 1 . Ambos foram adaptados para a populaçáo brasileira e já foram utilizados em estudos anteriores; 2 . Ambos medem, em anos e meses, áreas importantes do desenvolvimento isoladamente, mas também oferecem uma medida global do desenvolvimento, o que permite comparar a criança avaliada com o que é esperado para a idade cronológica; 3. Permitem avaliar os efeitos da intervenção no desenvolvimento das crianças, em áreas específicas e no desenvolvimento como um todo.

O PEP-R avalia tanto atraso no desenvolvimento como comportamentos típicos de autismo e oferece informaçóes sobre sete áreas na Escala de Desenvolvimento: imitação, percepção, coordenação motora fina, coordenação motora grossa, integração olho mão, desenvolvimento cognitivo e cognitivo verbal, além de quatro áreas na Escala de Comportamento: linguagem, relacionamento e afeto, respostas sensoriais e interesses por materiais. Pode ser utilizado com crianças de 6 meses a 12 anos de idade. Foi adaptado e validado para a populaçấo brasileira (LEON et al., 2004). Nesse estudo foram utilizados os dados da Escala de Desenvolvimento (não os da Escala de Comportamento).

O IPO avalia o padrão de desenvolvimento infantil em cinco áreas: linguagem, socialização, desenvolvimento motor, cognição e autocuidados, em períodos de idade que vão de 0 a 6 anos. Não é um instrumento destinado a avaliar especificamente o desenvolvimento de crianças com autismo, mas avalia o desenvolvimento de qualquer criança, independente do diagnóstico. O inventário foi adaptado e operacionalizado para a população brasileira (WILLIAMS; AIELLO, 2001). 


\subsection{Procedimentos}

Inicialmente cada criança foi avaliada (pré-testes) por dois profissionais do CEI com formação em psicologia ou em terapia ocupacional. A aplicação do PEP-R ocorreu em uma sala com poucos estímulos, em situação na qual um terapeuta apresentava as atividades e o outro registrava o desempenho da criança. O IPO foi aplicado na residência das crianças, em situação mais próxima do natural, a partir da observação do comportamento da criança e do relato dos cuidadores. É importante ressaltar que algumas habilidades são medidas pelos dois instrumentos, porém de maneiras distintas e com critérios diferentes. A aplicação do PEP-R foi feita em ambiente artificial (sala com poucos estímulos) e sob demanda (o avaliador solicitava que a criança fizesse a atividade); a aplicação do IPO foi realizada em ambiente natural (residência dos participantes) por meio da observação e do relato, sem demanda direta. Foram necessárias, em média, duas semanas para a aplicação dos instrumentos de avaliação, análise dos dados e redação de um relatório com os resultados. A avaliação final (pós-testes) foi realizada entre 8 e 13 meses (ver Tabela 1) após a avaliação inicial, em situaçóes semelhantes à inicial; PEP-R aplicado em uma sala com poucos estímulos e O IPO aplicado na residência das crianças. Nem sempre a mesma dupla de terapeutas realizava a avaliação inicial e a final. Após a avaliação inicial começava-se a intervenção.

A intervenção foi realizada na residência das crianças com autismo, por meio da capacitação dos cuidadores. Dois profissionais, um psicólogo analista do comportamento e um terapeuta ocupacional, estavam presentes na casa dos participantes, duas vezes por semana (cada profissional uma vez por semana), em sessóes de uma hora cada. A função dos profissionais era ensinar os cuidadores a realizar as atividades com as crianças com autismo. Além disso, os profissionais também ensinavam os cuidadores a fazer registros sistemáticos do desempenho das crianças nas atividades (em protocolos elaborados pelo CEI, específicos para cada atividade) e supervisionavam a realização correta das atividades e dos registros para garantir a fidedignidade da intervenção.

Para cada criança era estabelecido um currículo de habilidades básicas, conforme Gomes e Silveira (2016), e um de autocuidados baseado no IPO (WILLIAMS; AIELLO, 2001). Os profissionais de psicologia eram responsáveis pela implementação de habilidades básicas e os de terapia ocupacional pelas de autocuidados. O currículo de habilidades básicas era dividido em cinco áreas: atenção, imitação, linguagem expressiva, linguagem receptiva e pré-acadêmica. Para cada área o CEI desenvolveu programas de ensino em número variado, que se encontram detalhadamente descritos em Gomes e Silveira (2016), de modo a favorecer sua replicabilidade. $\mathrm{O}$ currículo de habilidades de autocuidados era dividido em 4 áreas: alimentação, higiene pessoal, vestuário e uso do banheiro, que englobavam habilidades variadas (ex. higiene pessoal: escovar os dentes, tomar banho, vestir e despir, entre outras).

O ensino das habilidades especificadas nos currículos de habilidades básicas e de autocuidados era realizado pelos cuidadores, com suas respectivas crianças com autismo, seguindo as orientações sistemáticas dos profissionais especializados. As orientaçóes abordavam tanto as condiçôes de ensino (a preparação do ambiente e de materiais, como apresentar materiais e instruções, a exigência de resposta ativa, a apresentação de consequências identificadas como potencialmente reforçadoras, os critérios para concluir cada tarefa ou cada programa, entre outros aspectos), como o manejo geral dos comportamentos da criança. Para cada programa 
eram especificados critérios de aprendizagem, uso de reforçadores, procedimentos de correção e número de habilidades treinadas por sessão.

Os cuidadores também registravam, em protocolos específicos para cada atividade, a data de realização da atividade, o número de tentativas de ensino e o desempenho da criança. Os protocolos utilizados eram simples (na maioria dos casos bastava marcar $\mathrm{x}$ para acertos ou erros) para facilitar o registro dos cuidadores (GOMES; SILVEIRA, 2016). Os procedimentos de ensino eram fundamentados em Análise do Comportamento, porém nenhuma capacitação teórica foi realizada com os cuidadores, apenas orientaçóes práticas e didáticas, com o uso preferencial pela modelação e uso mínimo de termos técnicos.

As atividades eram realizadas pelos cuidadores, no geral, 5 vezes por semana, 3 horas por dia ( 2 horas de atividades de habilidades básicas e 1 hora de autocuidados), totalizando 15 horas semanais de estimulação para cada criança. Mais de um programa de ensino do currículo de habilidades básicas era inserido simultaneamente, dependendo da disponibilidade do cuidador e da criança e seguindo os critérios descritos por Gomes e Silveira (2016). Em relação ao currículo de autocuidados, todas as habilidades eram ensinadas simultaneamente ao longo de todo o período de intervenção, sempre articuladas com as situaçóes de vida diária nas quais deveriam ocorrer.

A coleta de dados foi retrospectiva, realizada por meio das avaliações aplicadas pelos profissionais do CEI e dos protocolos de registro das atividades, preenchidos pelos cuidadores (que se referiam às atividades que eles realizavam com as crianças). Isto é, os pesquisadores trabalharam com os dados cujos registros foram realizados pelos profissionais do CEI e pelos cuidadores. A coleta e análise dos dados foram realizadas entre janeiro de 2015 e novembro de 2016.

Os procedimentos empregados nesse estudo foram aprovados pelo Comitê de Ética da Faculdade de Ciências Médicas de Minas Gerais (Parecer número 923.913).

Dois avaliadores independentes e ingênuos quanto ao propósito da pesquisa contabilizaram avaliaçóes (realizadas pelos profissionais do CEI) e protocolos de registro das atividades (preenchidos pelos cuidadores) de 3 crianças selecionadas por sorteio (aproximadamente 30\% do total) e o coeficiente de concordância (Kazdin, 1982) foi de 87,5\%.

\section{Resultados}

As Figuras 1 e 2 apresentam os resultados individuais dos participantes com autismo no PEP-R e no IPO, por área do desenvolvimento e na somatória de todas as áreas. Os dois instrumentos apresentam pontuaçóes específicas para cada idade cronológica; a criança está de acordo com o que é esperado para a idade cronológica quando obtém pontuação compatível com a pontuação determinada para aquela idade; pontuação abaixo da determinada indica atraso no desenvolvimento.

O PEP-R avalia sete áreas na Escala de Desenvolvimento (Figura1): imitação, percepção, coordenação motora fina, coordenação motora grossa, integração olho mão, desenvolvimento cognitivo e cognitivo verbal. A soma da pontuação das sete áreas oferece uma medida total do desenvolvimento (Pontuação do Desenvolvimento). 
Na área de imitação P1, P2 e P7, obtiveram pontuação inicial (pré) compatível com o que era esperado para a idade cronológica e ganharam habilidades após a intervenção, ficando acima do que era esperado para a idade cronológica na avaliação final (pós). Os outros participantes obtiveram pontuação abaixo do que era esperado para a idade cronológica na avaliação inicial e ganharam habilidades após a intervenção, porém apenas P5 e P9 ficaram de acordo com o que era esperado para a idade cronológica na avaliação final.

Na percepção, P7 obteve pontuação compatível com o que era esperado para a idade cronológica e ganhou habilidades após a intervenção, ficando acima do que era esperado na avaliação final. Os outros participantes obtiveram pontuação abaixo do que era esperado na avaliação inicial e ganharam habilidades, porém apenas P1, P2, P5 e P9 ficaram de acordo com o que era o esperado para a idade cronológica na avaliação final.

Nas áreas de coordenação motora fina e de coordenação motora grossa, P1 e P2 obtiveram pontuação compatível com o que era esperado para a idade cronológica e melhoram após a intervenção, ficando acima do que era esperado para a idade na avaliação final. Os outros participantes obtiveram pontuação abaixo e ganharam habilidades após a intervenção, porém apenas P5 e P7 ficaram de acordo com o que era esperado para a idade cronológica na avaliação final de coordenação motora fina e P7 na de coordenação motora grossa.

$\mathrm{Na}$ integração olho mão, P1 obteve pontuação compatível com o que era esperado para a idade cronológica na avaliação inicial e ganhou habilidades após a intervenção, ficando acima do que era esperado para a idade na avaliação final. Os outros participantes obtiveram pontuação abaixo do que era esperado para a idade na avaliação inicial, ganharam habilidades com a intervenção, porém apenas P2, P4 e P7 ficaram de acordo com o que era esperado para a idade cronológica na avaliação final.

$\mathrm{Na}$ área de desenvolvimento cognitivo, nenhum participante apresentou pontuação compatível com o que era esperado para a idade cronológica na avaliação inicial e todos ganharam habilidades após a intervenção, porém apenas P1, P2 e P7 ficaram de acordo com o que era esperado para a idade cronológica na avaliação final.

Na área de cognitivo verbal, P1 e P2 obtiveram pontuação compatível com o que era esperado para a idade cronológica na avaliação inicial e ganharam habilidades após a intervenção, ficando acima do que era esperado na avaliação final. Em relação aos outros participantes, apenas P6, P7 e P9 ganharam habilidades após a intervenção e somente P7 obteve pontuação de acordo com o que era esperado para a idade cronológica na avaliação final.

A Pontuação do Desenvolvimento indicou que P1 e P2 obtiveram pontuação compatível com o que era esperado para a idade cronológica na avaliação inicial e melhoram após a intervenção, ficando acima do que era esperado na avaliação final. Os outros participantes obtiveram pontuação abaixo do que era esperado para a idade cronológica e ganharam habilidades após a intervenção, porém apenas P7 apresentou pontuação compatível com o que era esperado para a idade cronológica na avaliação final.

Os participantes ganharam habilidades em todas as áreas, porém esses ganhos ocorreram de maneira variada. Desempenhos mais baixos foram observados nas áreas de desempenho cognitivo e cognitivo verbal, provavelmente porque essas áreas englobam habilidades mais 
complexas que estão diretamente relacionadas ao repertório de fala e que precisariam de mais tempo para sem refinadas, além do primeiro ano de intervenção. Os participantes mais novos (P1 e P2) e o único participante que apresentava fala antes do início da intervenção (P7) obtiveram os melhores resultados. Os participantes que não apresentaram fala após a intervenção (P3, P4, P6) obtiveram, no geral, as pontuaçôes mais baixas nas avaliaçóes iniciais e finais (exceto P4 em integração olho-mão).

O IPO avalia cinco áreas (Figura 2): linguagem, socialização, desenvolvimento motor, cognição e autocuidados. A soma da pontuação das cinco áreas oferece uma medida total do desenvolvimento (Global).

Nas áreas de autocuidados e de linguagem, todos os participantes apresentaram pontuação abaixo do que a esperada para a idade cronológica na avaliação inicial e ganharam habilidades com a intervenção, porém apenas P1, P2 e P5 obtiveram pontuação compatível ao que era esperado para a idade cronológica na avaliação final em autocuidados e P2 em linguagem.

Na área de cognição P1 e P2 obtiveram pontuação compatível com o que era esperado para a idade cronológica na avaliação inicial e ganharam habilidades após a intervenção, porém apenas P2 ficou com pontuação acima do que era esperado para a idade cronológica na avaliação final. Os outros participantes obtiveram pontuaçáo inicial abaixo do que era esperado para a idade cronológica e ganharam habilidades após a intervenção, porém apenas P7 ficou com pontuação compatível ao que era esperado para a idade cronológica na avaliação final.

$\mathrm{Na}$ área de desenvolvimento motor, todos os participantes obtiveram pontuação compatível ou bem próximo ao que era esperado para a idade cronológica e melhoram pontuação após a intervenção. Porém, apenas P1, P2, P5, P7, P8 obtiveram pontuação compatível ao que era esperado para a idade cronológica na avaliação final.

Na socialização P7 obteve pontuação inicial compatível com o que era esperado para a idade cronológica, ganhou habilidades com a intervençáo, porém apresentou pontuação abaixo do que era esperado para a idade cronológica na avaliação final. Os outros participantes obtiveram pontuação inicial abaixo do que era esperado para a idade, ganharam habilidades com a intervenção, porém apenas P1 e P2 ficaram com pontuação de acordo com o que era esperado para a idade cronológica na avaliação final.

A medida global do desenvolvimento indicou que todos os participantes obtiveram pontuação abaixo do que era esperado para a idade cronológica na avaliação inicial, apresentaram ganhos com a intervenção, porém apenas P2 apresentou pontuação de acordo com o que era esperado para a idade cronológica na avaliação final.

Os resultados gerais do IPO são semelhantes aos do PEP-R; os participantes ganharam habilidades em todas as áreas, de maneira variada, com desempenhos mais baixos em linguagem. Os participantes P1, P2 e P7 também obtiveram os melhores resultados. 

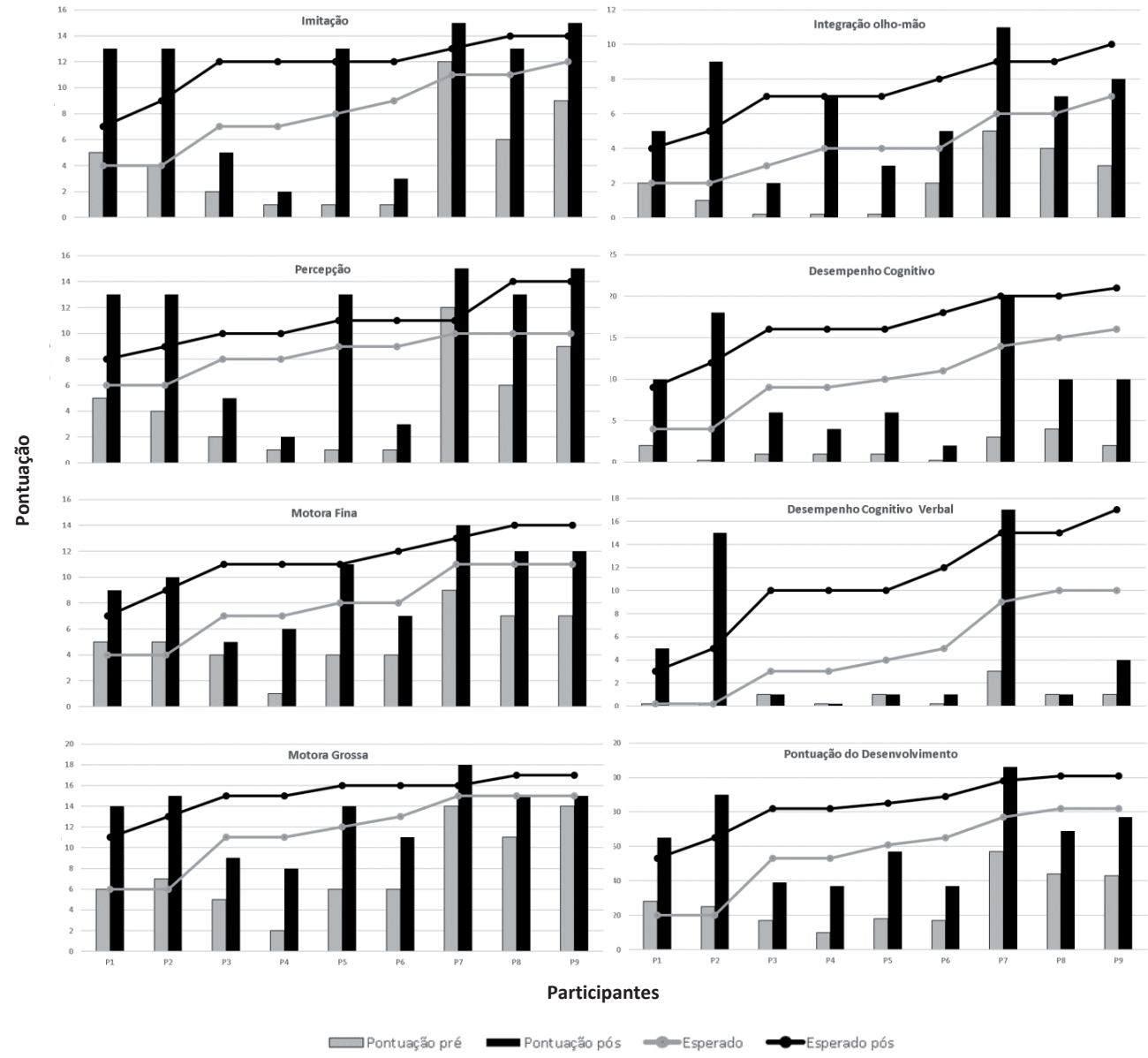

Figura 1- Resultados individuais dos participantes com autismo no PEP-R por área do desenvolvimento e na somatória de todas as áreas. Linha cinza clara indica resultado esperado para a idade cronológica na avaliação inicial (pré); linha cinza escura indica resultado esperado para a idade cronológica na avaliação final (pós); barra cinza clara indica resultado de cada participante na avaliação inicial (pré); barra cinza escura indica resultado de cada participante na avaliação final (pós).

Fonte: elaboração própria. 


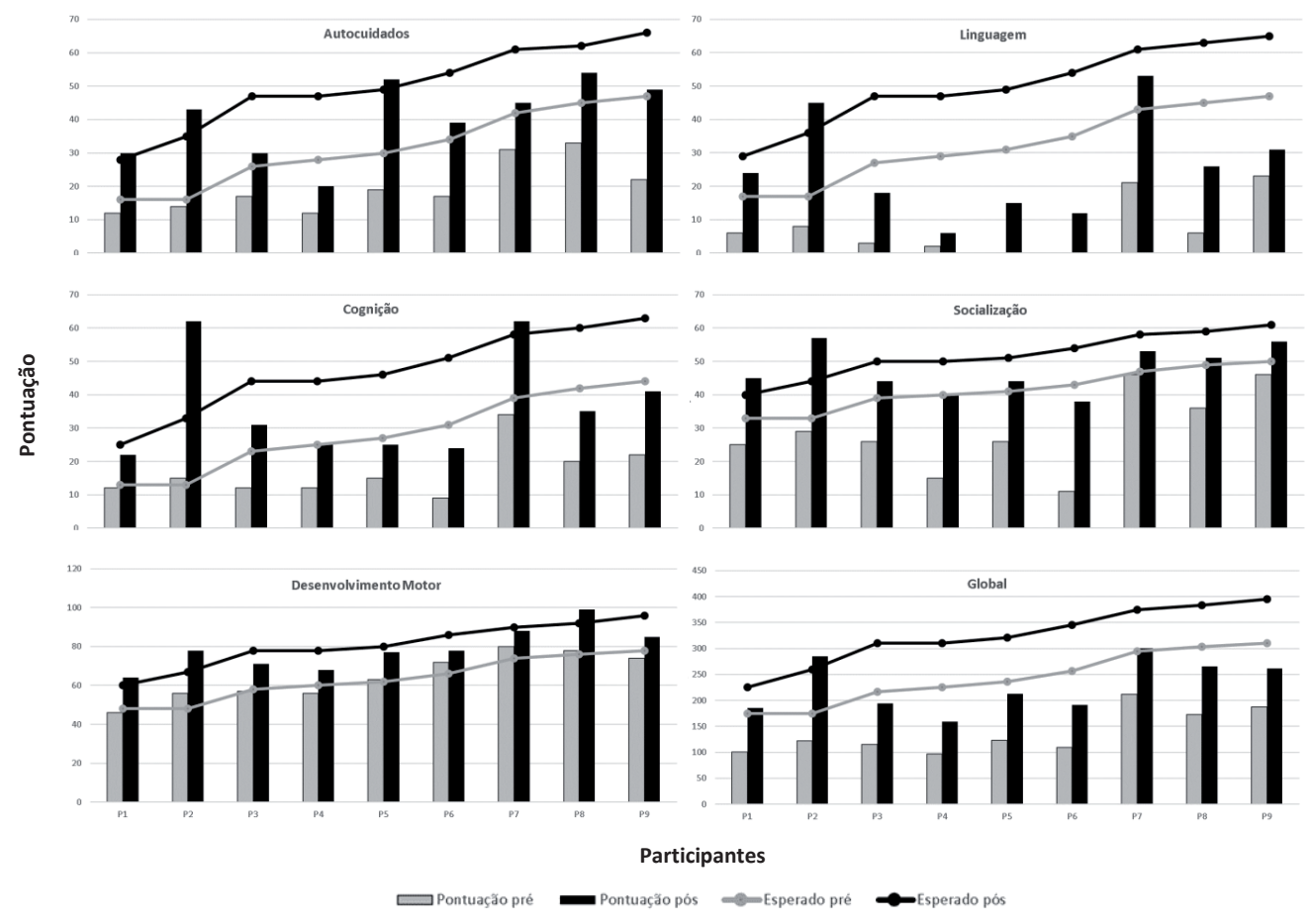

Figura 2- Resultados individuais dos participantes com autismo no IPO por área do desenvolvimento e na somatória de todas as áreas. Linha cinza clara indica resultado esperado para a idade cronológica na avaliaçáo inicial (pré); linha cinza escura indica resultado esperado para a idade cronológica na avaliação final (pós); barra cinza clara indica resultado de cada participante na avaliação inicial (pré); barra cinza escura indica resultado de cada participante na avaliação final (pós).

Fonte: elaboração própria.

\section{Discussóes E CONCLUSÓES}

O objetivo desse estudo, baseado em dados retrospectivos do primeiro ano de Intervenção Comportamental Intensiva, foi avaliar os efeitos desse tipo de intervenção no desenvolvimento de crianças brasileiras com autismo, realizada precocemente por meio da capacitação de cuidadores, em ambiente domiciliar. Dois instrumentos padronizados (PEP-R e IPO) foram utilizados, antes e após a intervenção, para medir o desenvolvimento dos participantes e avaliar possíveis ganhos decorrentes do processo.

A literatura indica de maneira consistente, por meio de dezenas de estudos publicados desde a década de 1980, que este tipo de intervenção pode promover ganhos significativos no desenvolvimento de crianças com autismo, embora a descrição da proporção dos ganhos seja variada (LOVAAS, 1987; SHERMAN et al., 1988; SMITH, 1999; WARREN et al., 2011). Por outro lado, a literatura também indica a necessidade de replicaçóes com delineamentos experimentais mais rigorosos, porém salienta que há aspectos que dificultam a realização desse tipo de pesquisa, como a duração longa (2 anos ou mais), o controle difícil de variáveis (muitas 
habilidades ensinadas simultaneamente, muitas horas de intervenção semanal e muitas pessoas envolvidas) e o alto custo de execução (BOYD; CORLEY, 2001; SMITH, 1999; WARREN et al., 2011; WEISS, 1999).

Segundo Smith e colaboradores (2000), as pesquisas existentes, combinadas com os diversos estudos de caso descrevendo os bons resultados da Intervenção Comportamental Intensiva (MAURICE, 1993), indicaram a viabilidade desse tipo de intervenção e criaram uma demanda alta das famílias de pessoas com autismo por esse tipo de tratamento. Essa demanda encontrou dois problemas práticos: o elevado número de crianças com autismo (que é maior do que a quantidade de profissionais capacitados) e o alto custo financeiro desse tipo de intervenção (em função da quantidade necessária de horas semanais de terapia). Os autores ainda enfatizaram que se tornou inviável utilizar profissionais especializados para realizar todo o tratamento e que a solução mais comum para implementar esse tipo de intervenção tem sido empregar cuidadores para realização das atividades, com orientação e supervisão de terapeutas comportamentais capacitados (ANDERSON et al., 1987; DAWSON et al., 2010; MCCOCHIE; DIGGLE, 2006, SHEINKOPF; SIELGEL, 1998; SHERMAN et al., 1988; SMITH et al., 2000), semelhante ao que foi realizado nesse estudo. Estudos que compararam os efeitos desse tipo de intervenção realizada por terapeutas ou cuidadores são escassos, porém essa pode ter sido uma variável que influenciou nos resultados e deve ser investigada em estudos posteriores.

De maneira geral, os dados desse estudo atual são condizentes com a literatura e indicaram ganhos no desenvolvimento dos participantes; todas as crianças obtiveram ganhos em todas as áreas do IPO e os dados do PEP-R são semelhantes, exceto pela área de desempenho cognitivo verbal, na qual quatro crianças (P3, P4, P5 e P8) não obtiveram ganhos. Ganhos mais expressivos parecem estar relacionados a algumas características da criança no início da intervenção. Nesse sentido, estudos que investigaram variáveis indicadoras de maiores ganhos no desenvolvimento em intervençóes comportamentais intensivas apontaram que crianças mais novas, com mais habilidades cognitivas e de linguagem obtiveram ganhos maiores (BEGLINGER; SMITH, 2005; GREEN, 1996; LUISELLI et al., 2000; HARRIS; HANDLEMAN, 2000). Os dados desse estudo são consistentes com essa literatura, pois os dois participantes mais novos (P1, P2) e o único participante que apresentava fala antes da intervenção (P7) obtiveram os melhores resultados; essas 3 crianças (P1, P2 e P7) ficaram com pontuação total final (Figura 1, Pontuação do Desenvolvimento) compatível com o que era esperado para a idade cronológica, de acordo com os critérios do PEP-R, e uma delas (P2) obteve pontuação total final (Figura 2, Global) compatível com o que era esperado para a idade cronológica de acordo com o IPO. Outro aspecto importante é que essas crianças se diferenciaram das outras ao obter ganhos mais robustos nas áreas de desempenho cognitivo e desempenho cognitivo verbal do PEP-R (Figura 1) e de linguagem do IPO (Figura 2).

Apesar de não terem ficado com pontuação de acordo com o que era esperado para idade cronológica na avaliação final, 5 crianças (P3, P4, P5, P8 e P9) ficaram com a pontuação total final próxima à que era esperada para a idade cronológica na avaliação inicial, de acordo com os critérios do PEP-R e 6 (P1, P3, P5, P7, P8 e P9), de acordo com os critérios do IPO. Além disso, 5 crianças (P1, P2, P5, P8, P9) do total de 8 apresentaram fala na avaliação final. Estes dados sugerem que a intervenção foi efetiva em promover ganhos de habilidades que as 
crianças não apresentavam e que deixavam o desenvolvimento delas em atraso, porém, como as crianças ficaram mais velhas, estes ganhos não foram suficientes para que elas conseguissem pontuação compatível com a esperada para a idade cronológica na avaliação final. Dessa maneira, apesar da intervenção ter promovido uma melhora no desenvolvimento dessas crianças, esta melhora não foi suficiente para superar o atraso no desenvolvimento.

Há a necessidade de continuidade do estudo para avaliar os efeitos de longo prazo, já que a literatura indica que intervençóes comportamentais intensivas devem ocorrer por dois anos ou mais e neste estudo foram analisados apenas os dados do primeiro ano de intervenção (GREEN, 1996). Além disso, há dados na literatura indicando que no primeiro ano deste tipo de intervenção os ganhos no desenvolvimento podem ser variados, conforme foi observado no estudo atual, com desempenhos mais expressivos em algumas áreas do que em outras (DAWSON et al., 2010). Possivelmente ganhos cognitivos e de linguagem devem ocorrer de maneira mais expressiva no segundo ano de intervenção intensiva, pois no primeiro ano a maioria das crianças ainda não falava e o foco do ensino foi em habilidades básicas como a imitação (GOMES; SILVEIRA, 2016).

Os resultados descritos neste estudo são condizentes com a literatura que indica ganhos no desenvolvimento de crianças com autismo, decorrentes desse tipo de intervenção, especialmente quando iniciada em idades abaixo de 48 meses (DAWSON et al., 2010, LOVAAS, 1987; SHERMAN et al., 1988; SMITH, 1999; WARREN et al., 2011). Por outro lado, o estudo indica a necessidade de novas investigações, com um número maior de participantes, com melhor controle experimental e que avaliem os efeitos de longo prazo desse tipo de intervenção.

\section{REFERÊNCIAS}

AMERICAN PSYCHIATRIC ASSOCIATION. Diagnostic and statistical manual of mental disorders DSM-5. Washington: APA, 2013.

AMERICAN PSYCHIATRIC ASSOCIATION. Manual diagnóstico e estatístico de transtornos mentais: texto revisado (DSM-IV-TR). Artmed, 2002.

ANDERSON, S. et al. Intensive home-based early intervention with autistic children. Education and Treatment of Children, p.352-366, 1987.

BAER, D.; WOLF, M.; RISLEY, T. Some still-current dimensions of applied behavior analysis. Journal of Applied Behavior Analysis, v.20, n.4, p.313-327, 1987.

BEGLINGER, L.; SMITH, T. Concurrent validity of social subtype and IQ after early intensive behavioral intervention in children with autism: a preliminary investigation. Journal of Autism and Developmental Disorders, v.35, n.3, p.295-303, 2005.

BOYD, R..; CORLEY, M. Outcome survey of early intensive behavioral intervention for young children with autism in a community setting. Autism, v.5, n.4, p.430-441, 2001.

CAMPBELL, M. et al. Treatment of autistic disorder. Journal of the American Academy of Child \& Adolescent Psychiatry, v.35, n.2, p.134-143, 1996.

DAWSON, G. et al. Randomized, controlled trial of an intervention for toddlers with autism: the Early Start Denver Model. Pediatrics, v.125, n.1, p. e17-e23, 2010. 
ELDEVIK, S. et al. Meta-analysis of early intensive behavioral intervention for children with autism. Journal of Clinical Child \& Adolescent Psychology, v.38, n.3, p.439-450, 2009.

GARGIULO, R. Persons with autism spectrum disorder. In: GARGIULO, R. (Org.). Special education in contemporary society: an introduction to exceptionality Belmont (CA): Wadsworth/Thomson Learning, 2006. p.521-561.

GREEN, G. Early behavioral intervention for autism: What does research tell us. In: MAURICE, C.; GREEN, G.; LUCE, S. (Org.). Behavioral intervention for young children with autism: a manual for parents and professionals. Pro-ed, 1996. p.29-44.

GOMES, C.; SILVEIRA, A. Ensino de habilidades básicas para pessoas com autismo: manual para Intervenção Comportamental Intensiva. Curitiba: Appris, 2016.

HARRIS, S.; HANDLEMAN, J. Age and IQ at intake as predictors of placement for young children with autism: A four-to six-year follow-up. Journal of Autism and Developmental Disorders, v.30, n.2, p.137-142, 2000.

LEON, V. et al. Propriedades psicométricas do Perfil Psicoeducacional Revisado: PEP-R. Avaliação Psicológica, v.3, n.1, p.39-52, 2004.

LOVAAS, O. Behavioral treatment and normal educational and intellectual functioning in young autistic children. Journal of Consulting and Clinical Psychology, v.55, n.1, p.3, 1987.

LUISELLI, J. et al. Home-based behavioral intervention for young children with autism/pervasive developmental disorder: a preliminary evaluation of outcome in relation to child age and intensity of service delivery. Autism, v.4, n.4, p.426-438, 2000.

MAURICE, C. Let me hear your voice. New York: Knopf, 1993.

MCCONACHIE, H.; DIGGLE, T. Parent implemented early intervention for young children with autism spectrum disorder: a systematic review. Journal of Evaluation in Clinical Practice, v.13, n.1, p.120-129, 2007.

MCEACHIN, J.; SMITH, T.; LOVAAS, I. Long-term outcome for children with autism who received early intensive behavioral treatment. American Journal of Mental Retardation, v.97, p.359-359, 1993.

ORGANIZAÇÃO MUNDIAL DE SAÚDE. Classificação de transtornos mentais e de comportamentos da CID-10: descriçóes clínicas e diretrizes diagnósticas. Porto Alegre: Artes Médicas, 1993.

SCHOPLER, E. et al. Individualized assessment of autistic and developmentally disabled children: Psychoeducational Profile Revised (PEP-R). Austin, TX: Pro-Ed, 1990.

SHEINKOPF, S.; SIEGEL, B. Home-based behavioral treatment of young children with autism. Journal of Autism and Developmental Disorders, v.28, n.1, p.15-23, 1998.

SHERMAN, J. et al. Treatment of autistic children: relative effectiveness of residential, out-patient and home-based interventions. Child Psychiatry and Human Development, v.19, n.2, p.109-125, 1988.

SKINNER, B. F. The technology of teaching. New York: Appleton-CenturyCrofts, 1968.

SMITH, T. Outcome of early intervention for children with autism. Clinical Psychology: Science and Practice, v.6, n.1, p.33-49, 1999.

SMITH, T.; BUCH, G.; GAMBY, T. Parent-directed, intensive early intervention for children with pervasive developmental disorder. Research in Developmental Disabilities, v.21, n.4, p.297-309, 2000. 
TODOROV, J.; HANNA, E. Análise do comportamento no Brasil. Psicologia: Teoria e Pesquisa, Brasília, v.26, n. 25ANOS, p.143-154, 2010.

VIRUÉS-ORTEGA, J. Applied behavior analytic intervention for autism in early childhood: Metaanalysis, meta-regression and dose-response meta-analysis of multiple outcomes. Clinical Psychology Review, v.30, n.4, p.387-399, 2010.

WARREN, Z. et al. A systematic review of early intensive intervention for autism spectrum disorders. Pediatrics, v.127, n.5, p. e1303-e1311, 2011.

WEISS, M. Differential rates of skill acquisition and outcomes of early intensive behavioral intervention for autism. Behavioral Interventions, v.14, n.1, p.3-22, 1999.

WILlIANS, L; AIELLO, A. Inventário Portage operacionalizado. São Paulo: Mennon, 2001. 